\begin{tabular}{c|c|c||c|c|c}
\hline$n$ & $\begin{array}{c}\text { First } \\
\text { Case: } \\
\text { Maximum } \\
\text { Number } \\
\text { of Real } \\
\text { Non- } \\
\text { Negative } \\
\text { Abscissae }\end{array}$ & $\begin{array}{c}\text { Second } \\
\text { Case: } \\
\text { Maximum } \\
\text { Number } \\
\text { of Real } \\
\text { Abscissae }\end{array}$ & $n$ & $\begin{array}{c}\text { First } \\
\text { Case: } \\
\text { Maximum } \\
\text { Number } \\
\text { of Real } \\
\text { Non- } \\
\text { Negative } \\
\text { Abscissae }\end{array}$ & $\begin{array}{c}\text { Second } \\
\text { Case: } \\
\text { Maximum } \\
\text { Number } \\
\text { of Real } \\
\text { Abscissae }\end{array}$ \\
\cline { 2 - 4 } 11 & 5 & 7 & 31 & 11 & 11 \\
12 & 7 & 6 & 32 & 11 & 10 \\
13 & 7 & 7 & 33 & 11 & 11 \\
14 & 7 & 6 & 34 & 13 & 10 \\
15 & 7 & 7 & 35 & 13 & 11 \\
16 & 7 & 6 & 36 & 13 & 10 \\
17 & 7 & 7 & 37 & 13 & 11 \\
18 & 9 & 6 & 38 & 13 & 14 \\
19 & 9 & 7 & 39 & 13 & 15 \\
20 & 9 & 10 & 40 & 13 & 14 \\
21 & 9 & 11 & 41 & 13 & 15 \\
22 & 9 & 10 & 42 & 13 & 14 \\
23 & 9 & 11 & 43 & 13 & 15 \\
24 & 9 & 10 & 44 & 15 & 14 \\
25 & 9 & 11 & 45 & 15 & 15 \\
26 & 11 & 10 & 46 & 15 & 14 \\
27 & 11 & 11 & 47 & 15 & 15 \\
28 & 11 & 10 & 48 & 15 & 14 \\
29 & 11 & 11 & 49 & 15 & 15 \\
30 & 11 & 10 & 50 & 15 & 14 \\
\hline
\end{tabular}

Battersea College of Technology London, S.W. 11, England

1. H. E. SALzER, "Equally weighted quadrature formulas over semi-infinite and infinite intervals," J. Math. Phys., v. 34, 1955, p. 54-63.

\title{
Search For Largest Polyhedra
}

\section{By Donald W. Grace}

The configuration of eight points on the unit sphere which determines the convex polyhedron of maximum volume is not known. It is not the cube, whose volume is $8 \sqrt{3} / 9$, or about 1.5396 , since the volume of the double pyramid (one point at each of the poles and the other six distributed uniformly around the equator) is $\sqrt{ } \overline{3}$, or about 1.732 . [1, page 7$]$. The purpose of the present work was to determine if an even larger polyhedron could be found.

The search was carried out using gradient methods on the Burroughs 220 computer. Each set of eight points on the sphere may be interpreted as a vector, $W$, in Euclidean 16-space. For each $W$, let $V(W)$ denote the volume of the convex hull of the eight points, and $G(W)$ the gradient of $V(W)$. A starting configuration was introduced into the computer and modified iteratively. At each iteration, the 16-dimensional vector, $W$, was replaced by $W^{\prime}=W+M \cdot G(W)$, for some real $M$

Received August 28, 1962. 
chosen so that $V\left(W^{\prime}\right)>V(W)$. Convergence was accelerated by letting the computer search for the multiplier, $M$, which caused the greatest increase in $V(W)$. at each iteration. This process was terminated when the magnitude of $G(W)$ became sufficiently small.

By use of Goldberg's conjecture [2, page 231] a polyhedron larger than the double pyramid was found. The coordinates of the final configuration, with the origin at the center of the sphere, are shown in the following table.

\begin{tabular}{c|r|r|r|r|r}
\hline \multirow{2}{*}{$\begin{array}{c}\text { Point } \\
\text { Number }\end{array}$} & \multicolumn{2}{|c|}{ Spherical Coordinates } & \multicolumn{3}{|c}{ Rectangular Coordinates } \\
\cline { 2 - 6 } & Longitude & Colatitude & $\mathrm{X}$ & $\mathrm{Y}$ & $\mathrm{Z}$ \\
\cline { 2 - 6 } & $-90^{\circ}$ & $34^{\circ} 41^{\prime}$ & 0 & -.569 & .822 \\
1 & $90^{\circ}$ & $104^{\circ} 5^{\prime}$ & 0 & .970 & -.243 \\
3 & $180^{\circ}$ & $75^{\circ} 55^{\prime}$ & -.970 & 0 & .243 \\
4 & $180^{\circ}$ & $145^{\circ} 19^{\prime}$ & -.569 & 0 & -.822 \\
5 & $-90^{\circ}$ & $104^{\circ} 5^{\prime}$ & 0 & -.970 & -.243 \\
6 & $0^{\circ}$ & $145^{\circ} 19^{\prime}$ & .569 & 0 & -.822 \\
7 & $0^{\circ}$ & $75^{\circ} 55^{\prime}$ & .970 & 0 & .243 \\
8 & $90^{\circ}$ & $34^{\circ} 41^{\prime}$ & 0 & .569 & .822 \\
\hline
\end{tabular}

The volume of this polyhedron is approximately 1.8157. This may be just another of the many relative maxima of the volume function, or it could be the absolute maximum.

It is known [3, page 127] that a polyhedron of maximal volume among all polyhedra with $n$ vertices inscribed in a sphere must be bounded solely by triangular faces. By Euler's relation, in any polyhedron with triangular faces and eight vertices the total number of edges is $3(8-2)=18$, so the average number of edges at each vertex is $2 \cdot 18 / 8=4.5$. In the polyhedron tabulated above, the number of edges at each vertex is either four or five, and is therefore as close as possible to the average. The dual of Goldberg's conjecture can be loosely stated as follows:

In the polyhedron of maximum volume formed by $n$ points on a sphere, the faces are triangular and the number of edges leading to any vertex is as nearly as possible equal to the average of such numbers.

This "medial polyhedron" conjecture is verified for those values of $n$ for which the largest convex hull is known.

Incidentally, the cube not only fails to have the maximum volume, it is not even a relative maximum. Introduction of the cube into the computer as the starting configuration resulted in the following larger polyhedron:

\begin{tabular}{c|c|c}
\hline Point & Longitude & Colatitude \\
\cline { 2 - 3 } 1 & 0 & $0^{\circ}$ \\
2 & 0 & $180^{\circ} 30^{\prime}$ \\
3 & 0 & $61^{\circ} 30^{\prime}$ \\
4 & 60 & $118^{\circ} 30^{\prime}$ \\
5 & 120 & $61^{\circ} 30^{\prime}$ \\
6 & 180 & $118^{\circ} 30^{\prime}$ \\
7 & 240 & $61^{\circ} 30^{\prime}$ \\
8 & 300 & $118^{\circ} 30^{\prime}$ \\
\hline
\end{tabular}




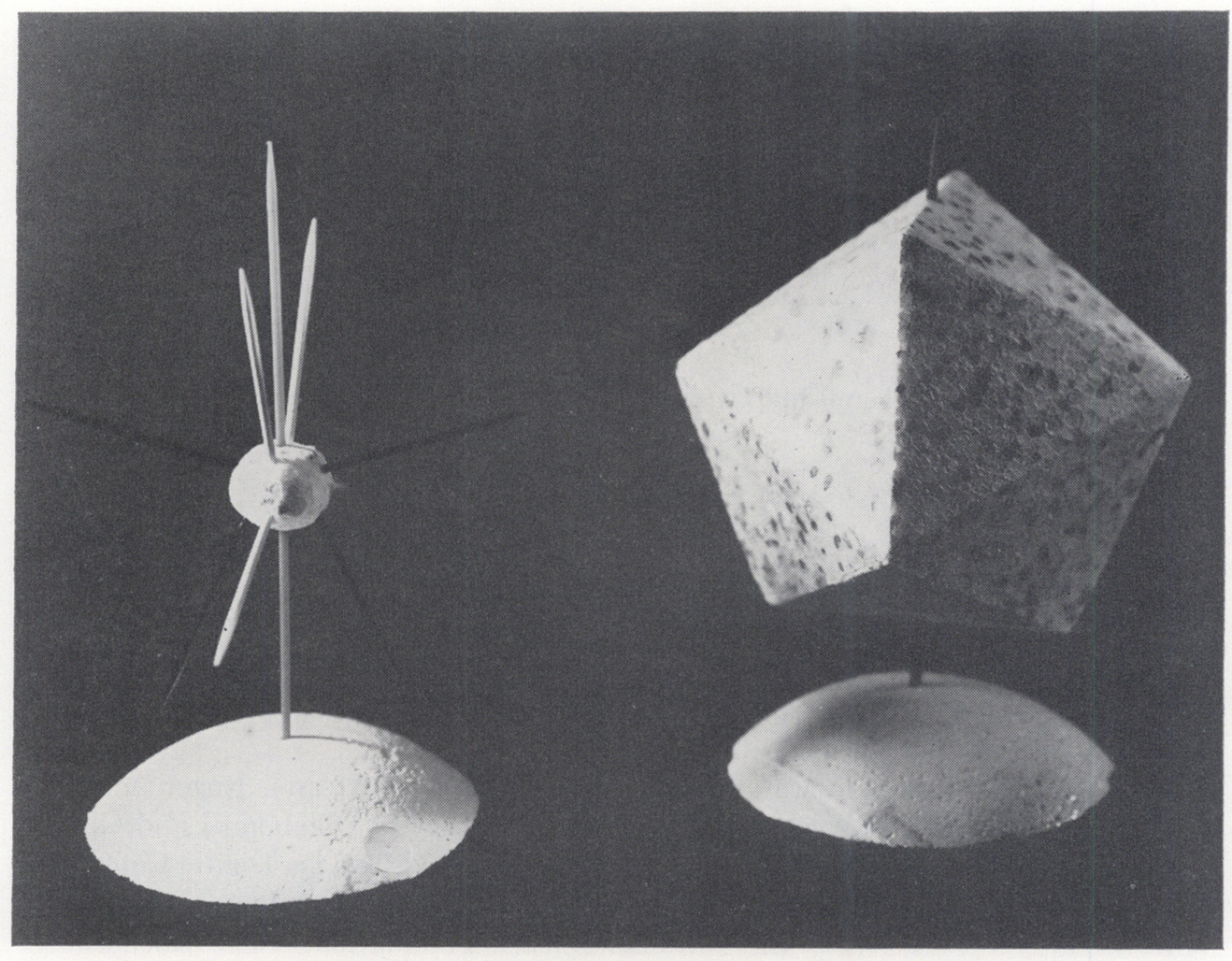

Fig. 1. The Largest Polyhedron Found.

The volume of this polyhedron is approximately 1.626 , compared with about 1.5396 for the cube.

The solid polyhedron in figure 1 is the largest one found for eight points on a sphere. The stick figure is the set of vectors which form the largest polyhedron. That is, the polyhedron is the convex hull of the stick figure.

The author wishes to thank Professor G. E. Forsythe of Stanford University's Computer Science Division for suggesting the problem, and Professor G. Pólya of Stanford's Mathematics Department for suggesting the duality of Goldberg's conjecture.

The referee notes that the locations of the vertices are close to the locations of the points of tangency of the eight faces of the largest known octahedron circumscribed about the sphere [2, pages 234 and 236].

The Procter and Gamble Company

Ivorydale Technical Center Cincinnati 17, Ohio

1. G. Pólya, "Mathematics as a subject for learning plausible reasoning," The Mathematics Teacher, v. 52, 1959.

2. Michael Goldberg, "The isoperimetric problem for polyhedra," Tôhoku Mathematical Journal, v. 40, April 1935, p. 226-236. 1953.

3. L. Fejes Tóth, Lagerungen in der Ebene auf der Kugel und im Raum, Springer-Verlag, 